University of Texas at El Paso

ScholarWorks@UTEP

$12-2013$

\title{
Finding the Best Function: A Way to Explain Calculus of Variations to Engineering and Science Students
}

Olga Kosheleva

The University of Texas at El Paso, olgak@utep.edu

Vladik Kreinovich

The University of Texas at El Paso, vladik@utep.edu

Follow this and additional works at: https://scholarworks.utep.edu/cs_techrep

Part of the Mathematics Commons

Comments:

Technical Report UTEP-CS-13-72

Published in Applied Mathematical Sciences, 2013, Vol. 7, No. 144, pp. 7187-7192.

\section{Recommended Citation}

Kosheleva, Olga and Kreinovich, Vladik, "Finding the Best Function: A Way to Explain Calculus of Variations to Engineering and Science Students" (2013). Departmental Technical Reports (CS). 803. https://scholarworks.utep.edu/cs_techrep/803 


\title{
Finding the Best Function: A Way to Explain Calculus of Variations to Engineering and Science Students
}

\author{
Olga Kosheleva ${ }^{1}$ and Vladik Kreinovich ${ }^{2}$ \\ ${ }^{1}$ Department of Teacher Education \\ ${ }^{2}$ Department of Computer Science \\ University of Texas at El Paso \\ $500 \mathrm{~W}$. University \\ El Paso, TX 79968, USA \\ olga@utep.edu,vladik@utep.edu
}

\begin{abstract}
In many practical problems, we need to find the most appropriate function: e.g., we need to find a control strategy $u(t)$ that leads to the best performance of a system, we need to find the shape of the car which leads to the smallest energy losses, etc. Optimization over an unknown function can be described by the known Euler-Lagrange equations. The traditional way of deriving Euler-Lagrange equations when explaining them to the engineering and science students is, however, somewhat over-complicated. We provide a new, simpler way to deriving these equations, a way in which we directly use the fact that when the optimum is attained, all partial derivatives are equal to 0 .
\end{abstract}

\section{Finding the Best Function: Euler-Lagrange Equations and How They Are Derived Now}

Optimization is ubiquitous. In practice, we often need to make choices. For example, in engineering, when we design an object (a car, a computer) or select a control (e.g., a route for the car or for a computer message), we need to select the values of several parameters describing the object or control.

Often, we can express our preferences by assigning, to each possible alternative, a numerical value describing the degree to which we are satisfied with this alternative. For example, when we design a high-performance computer, this satisfaction-describing numerical value is the throughput - the number of operations per second that this computer can perform. In such situations, we need to select the values of the corresponding parameters $x_{1}, \ldots, x_{n}$ for which the corresponding numerical value $F\left(x_{1}, \ldots, x_{n}\right)$ is the largest possible. 
One way to solve this optimization problem is to use the fact that in the optimal solution (at which the maximum is attained), all the partial derivatives are equal to 0 : $\frac{\partial F}{\partial x_{i}}=0$ for $i=1, \ldots, n$. This fact allows us to reduce the optimization problem to the problem of solving a system of $n$ equations with $n$ unknowns, a problem for which many algorithms are known.

Often, we need to find the best function. In many practical situations, it is sufficient to fix the values of finitely many parameters to describe an alternative. In many other situations, we need to select a function of one or several variables. For example, in control, we need to describe what control value $u(t)$ to apply at different moments of time $t$; when designing a ship, we need to describe its shape, etc. In such situations, instead of finitely many unknown values $x_{1}, \ldots, x_{n}$, we have an unknown function $f(t)$ (or $\left.f\left(x_{1}, \ldots, x_{n}\right)\right)$, and we need to find the "best" function.

A similar optimization problem is useful in physics, since it is known that many physical laws can be described as stating that the actual physical trajectories and/or actual fields minimizing an appropriate value (called "action"); see, e.g., [1]). For example, Newton's law $m \cdot \ddot{x}_{i}=-\frac{\partial V}{\partial x_{i}}$ that describes the motion of a particle with mass $m$ in a potential field $V(x)$ can be equivalently reformulated as minimizing the action functional $\int\left(\sum_{i=1}^{3} \frac{m \cdot\left(\dot{x}_{i}\right)^{2}}{2}-V\right) d t$.

Finding the best function: precise formulation of the problem. In general, for a single function of one variable $f(t)$, we need to optimize the value

$$
J \stackrel{\text { def }}{=} \int F(f(t), \dot{f}(t), t) d t \text {. }
$$

For a function $f\left(x_{1}, \ldots, x_{n}\right)$ of several variables, we need to optimize the value

$$
\int F\left(f\left(x_{1}, \ldots, x_{n}\right), f_{, 1}\left(x_{1}, \ldots, x_{n}\right), \ldots, f_{, n}\left(x_{1}, \ldots, x_{n}\right), x_{1}, \ldots, x_{n}\right) d x_{1} \ldots d x_{n},
$$

where $f_{, i} \stackrel{\text { def }}{=} \frac{\partial f}{\partial x_{i}}$. Similar formulations can be described for situations when several functions are needed to describe an alternative.

How to find the best function: Euler-Lagrange equations. It is known that the optimization problem of finding the best function can also be reduced to a system of equations - in this case, to a system of differential equations. The corresponding equations are known as Euler-Lagrange equation. For a function of one variable, these equations take the form

$$
\frac{\partial F}{\partial f}-\frac{d}{d t}\left(\frac{\partial F}{\partial \dot{f}}\right)=0
$$


For a function of several variables, these equations take the form

$$
\frac{\partial F}{\partial f}-\sum_{i=1}^{n} \frac{\partial}{\partial x_{i}}\left(\frac{\partial F}{\partial f_{, i}}\right)=0 .
$$

The corresponding equations are also known as calculus of variations; see, e.g., [2].

For example, for the 1-dimensional Newton's law, we have

$$
F(x, \dot{x}, t)=\frac{m \cdot(\dot{x})^{2}}{2}-V(x(t)) .
$$

Here, $\frac{\partial F}{\partial x}=-\frac{\partial V}{\partial x}$ and $\frac{\partial F}{\partial \dot{x}}=m \cdot \dot{x}$, so the Euler-Lagrange equation takes the form $-\frac{\partial V}{\partial x}-\frac{d}{d t}(m \cdot \dot{x})$, i.e., the desired form $m \cdot \ddot{x}=-\frac{\partial V}{\partial x}$.

How Euler-Lagrange equations are explained now. A usual way to derive Euler-Lagrange equations is to substitute functions of the type $f(t)=$ $f_{\text {opt }}(t)+\alpha \cdot g(t)$ into the functional $J$, where $f_{\text {opt }}(t)$ is the (unknown) optimal function and $g(t)$ is an arbitrary function. Since the functional $J$ attains its optimum when $f(t)=f_{\text {opt }}(t)$, i.e., when $\alpha=0$, we should get $\frac{d J}{d \alpha}=0$, i.e., $\int\left(\frac{\partial F}{\partial f} \cdot g(t)+\frac{\partial F}{\partial \dot{f}} \cdot \dot{g}(t)\right) d t=0$. Integrating by parts, we reduce this equation to $\int h(t) \cdot g(t) d t=0$, where $h(t) \stackrel{\text { def }}{=} \frac{\partial F}{\partial f}-\frac{d}{d t}\left(\frac{\partial F}{\partial \dot{f}}\right)$. This equality is true for every function $g(t)$, in particular, for a function $g(t)$ which is positive in a small vicinity of a point $t_{0}$ and equal to 0 everywhere else. For such a function $g(t)$, we get $\int h(t) \cdot g(t) d t \approx$ const $\cdot h\left(t_{0}\right)$, so we conclude that $h\left(t_{0}\right)=0$. Since this is true for every possible $t_{0}$, this means that $h(t)=0$ for all $t$. This is exactly Euler-Lagrange equations.

Pedagogical problem. For many engineering and science students, the above derivation is somewhat over-complicated: it requires differentiation under the integral, integration by part, and then an argument about $g(t)$. It goes way beyond their usual idea of simply equating partial derivatives to 0 . They sometimes ask a question: why cannot we just differentiate with respect to $f(t)$ and equate the derivatives to 0 ?

What we do in this paper. In this paper, we show that this is indeed possible to derive Euler-Lagrange equations by simply differentiating the objective function relative to the unknown and equating the derivatives to 0 .

This new derivations is not a complete proof (although it can be transformed into a proof), but it provides an explanation on the same level of rigor as the current explanation. 


\section{A New Way of Deriving Euler-Lagrange Equa- tions}

First step: reformulating the original problem. Formally, a derivative $\dot{f}(t)$ is defined as a limit $\lim _{\Delta t \rightarrow 0} \frac{f(t+\Delta t)-f(t)}{\Delta t}$. From the practical viewpoint, this means that when the value $\Delta t$ is very small, the derivative is practically equal to the above ratio: $\dot{f}(t) \approx \frac{f(t+\Delta t)-f(t)}{\Delta t}$. Thus,

$$
g(t) \stackrel{\text { def }}{=} F(f(t), \dot{f}(t), t) \approx F\left(f(t), \frac{f(t+\Delta t)-f(t)}{\Delta t}, t\right) .
$$

In particular, for the values $t_{0}, t_{1}=t_{0}+\Delta t, \ldots, t_{i}=t_{0}+i \cdot \Delta t, \ldots$, we get $g\left(t_{i}\right) \approx F\left(f_{i}, \frac{f_{i+1}-f_{i}}{\Delta t}, t_{i}\right)$, where we denoted $f_{i} \stackrel{\text { def }}{=} f\left(t_{i}\right)$

Similarly, an integral $J=\int g(t) d t$ can be defined as a limit of integral sums $\sum g\left(t_{i}\right) \cdot \Delta t$. This means that when $\Delta t$ is small, the integral $J$ is practically equal to the corresponding integral sum. From this viewpoint, if we select a very small $\Delta t$, then the optimized integral $J$ takes the form

$$
J \approx J_{0} \stackrel{\text { def }}{=} \sum_{i} F\left(f_{i}, \frac{f_{i+1}-f_{i}}{\Delta t}, t_{i}\right) \cdot \Delta t .
$$

In this approximation, the function $f(t)$ is described by its values $f_{i}=f\left(t_{i}\right)$. Thus, we need to find the values $f_{i}$ that maximize the above expression $J_{0}$.

Differentiating $J_{0}$ and equating derivative to 0 . To find the values $f_{i}$ that optimize the above expression $J_{0}$, let us differentiate $J_{0}$ with respect to $f_{i}$ and equate the corresponding partial derivative to 0 .

The derivative of the sum $J_{0}$ is equal to the sum of the derivatives. Each $i$-th term in the sum depends on two values $f_{i}$ and $f_{i+1}$. Thus, for each $i$, only two terms in the sum $J_{0}$ depend on $f_{i}$ : the $i$-th term $F\left(f_{i}, \frac{f_{i+1}-f_{i}}{\Delta t}, t_{i}\right) \cdot \Delta t$ and the $(i-1)$-st term $F\left(f_{i-1}, \frac{f_{i}-f_{i-1}}{\Delta t}, t_{i-1}\right) \cdot \Delta t$. Using the chain rule to differentiate both terms, we get

$$
\left(\frac{\partial F}{\partial f(t)}-\frac{\partial F}{\partial \dot{f}(t)}\left(t_{i}\right) \cdot \frac{1}{\Delta t}+\frac{\partial F}{\partial \dot{f}(t)}\left(t_{i-1}\right) \cdot \frac{1}{\Delta t}\right) \cdot \Delta t=0
$$

i.e.,

$$
\frac{\partial F}{\partial f(t)}-\frac{\partial F}{\partial \dot{f}(t)}\left(t_{i}\right) \cdot \frac{1}{\Delta t}+\frac{\partial F}{\partial \dot{f}(t)}\left(t_{i-1}\right) \cdot \frac{1}{\Delta t}=0 .
$$

The last two terms in the left-hand side of the formula (4) have a common factor 
$\frac{1}{\Delta t}$, so they can be reformulated as

$$
-\frac{\frac{\partial F}{\partial \dot{f}(t)}\left(t_{i}\right)-\frac{\partial F}{\partial \dot{f}(t)}\left(t_{i-1}\right)}{\Delta t}
$$

i.e., as $-\frac{h\left(t_{i}\right)-h\left(t_{i-1}\right)}{\Delta t}$, for $h(t) \stackrel{\text { def }}{=} \frac{\partial F}{\partial \dot{f}(t)}$. We have already mentioned that for small $\Delta t$, the ratio $\frac{h\left(t_{i}\right)-h\left(t_{i-1}\right)}{\Delta t}$ is practically equal to the derivative $\frac{d h(t)}{d t}$. Thus, the equation (4) takes the form

$$
\frac{\partial F}{\partial f(t)}-\frac{d}{d t}\left(\frac{\partial F}{\partial \dot{f}(t)}\right)=0
$$

Thus, we have derived the desired Euler-Lagrange equation (1).

Comment. A similar derivation can be done when $f$ is a function of several variables. In this case, we need to consider values $x_{i k}=x_{i 0}+k \cdot \Delta$ and take

$$
\begin{gathered}
f_{, i}=\frac{\partial f}{\partial x_{i}} \approx \\
\frac{f\left(x_{1}, \ldots, x_{i-1}, x_{i}+\Delta, x_{i+1}, \ldots, x_{n}\right)-f\left(x_{1}, \ldots, x_{i-1}, x_{i}, x_{i+1}, \ldots, x_{n}\right)}{\Delta} .
\end{gathered}
$$

\section{Case of Optimal Control}

Optimal control: formulation of the problem. In optimal control, we need to find the control $u(t)$ that maximized the objective function

$$
J=\int f(x(t), u(t), t) d t
$$

provided that we know the differential equation

$$
\dot{x}=g(x, u, t)
$$

that describes how the system reacts to a control.

One of the most widely used approaches to solve the optimal control. One of the main techniques for solving the optimal control problem is the Hamiltonian method, where we introduce an auxiliary function $\lambda(t)$ and solve the system consisting of two differential equations (6) and

$$
\dot{\lambda}=-\frac{\partial H}{\partial x},
$$


where $H(x, u, t) \stackrel{\text { def }}{=} f(x, u, t)+\lambda \cdot g(x, u, t)$, and an equation

$$
\frac{\partial H}{\partial u}=0
$$

From equation (8), we can express (at least implicitly) $u$ in terms of $x$ and $\lambda$. Substituting this expression for $u$ into equations (6) and (7), we get a system of two differential equations for two unknown functions $x(t)$ and $\lambda(t)$. Once we solve this system, we can then use (8) to find appropriate control values $\lambda(t)$.

Similar equations hold for the case when we have several variables $x=$ $\left(x_{1}, \ldots, x_{n}\right)$ describing the system and several variable $u=\left(u_{1}, \ldots, u_{l}\right)$ describing control. In this case, the auxiliary function $\lambda(t)$ also has $n$ components $\lambda_{1}(t), \ldots, \lambda_{n}(t)$; see, e.g., [3].

Usually, no derivation of this method is given to engineering and science students. In contrast to Euler-Lagrange equations which are several hundred years old, the Hamiltonian approach to optimal control is only a few decades old. As a result, it has not been pedagogically well-analyzed and wellpresented. Since no engineering-student-accessible derivation is available, this approach is usually presented to the engineering and science students without any derivations.

What we do. We show that this method can also be described to students whose only math background is basic calculus (i.e., basic limits, differentiation, optimization under constraints, and integration).

Similarly to the case of Euler-Lagrange equations, we illustrate this derivation on the simplest case, when both the state and the control are described by a single variable, i.e., when both $x(t)$ and $u(t)$ are scalars; however, the exact same derivation works for the case when we have several components of $x(t)$ and of $u(t)$.

Derivation. Similarly to the derivation of the Euler-Lagrange equations, from the practical viewpoint, the objective function (5) takes the form

$$
J \approx J_{0} \stackrel{\text { def }}{=} \sum_{i} f\left(x_{i}, u_{i}, t_{i}\right) \cdot \Delta t
$$

where we denoted $x_{i} \stackrel{\text { def }}{=} x\left(t_{i}\right)$ and $u_{i} \stackrel{\text { def }}{=} u\left(t_{i}\right)$. The equation (6) takes the form

$$
\frac{x_{i+1}-x_{i}}{\Delta t}-g\left(x_{i}, u_{i}, t_{i}\right)=0,
$$

or, equivalently,

$$
x_{i}+g\left(x_{i}, u_{i}, t_{i}\right) \cdot \Delta t-x_{i+1}=0 .
$$

The problem of finding the optimal control is thus reformulated as a typical problem of optimization under constraints: namely, we need to optimize the 
objective function (9) under the constraints (11). The usual tool for solving constraint optimization problems is the Lagrange multiplier approach, according to which the optimization of a function (9) under constraints (11) is equivalent to an unconstrained optimization of an auxiliary function

$$
h \stackrel{\text { def }}{=} \sum_{i} f\left(x_{i}, u_{i}, t_{i}\right) \cdot \Delta t+\sum_{i} \lambda_{i} \cdot\left(x_{i}+g\left(x_{i}, u_{i}, t_{i}\right) \cdot \Delta t-x_{i+1}\right),
$$

where $\lambda_{i}$ are Lagrange multipliers.

Differentiating the new objective function $h$ with respect to $u_{i}$ and equating derivative to 0 , we conclude that

$$
\frac{\partial f\left(x_{i}, u_{i}, t_{i}\right)}{\partial u_{i}} \cdot \Delta t+\lambda_{i} \cdot \frac{\partial g\left(x_{i}, u_{i}, x_{i}\right)}{\partial u_{i}} \cdot \Delta t=0 .
$$

Dividing both sides of this equality by $\Delta t$ and taking into account the above definition of $H(x, u, t)=f(x, u, t)+\lambda(t) \cdot g(x, u, t)$, we conclude that

$$
\frac{\partial H\left(f\left(x_{i}, u_{i}, t_{i}\right)\right.}{\partial u_{i}}=0 .
$$

This is exactly the desired equation (8).

Differentiating the function $h$ with respect to $x_{i}$, we must take into account that $x_{i}$ occurs both in the $i$-th and in the $(i-1)$-st terms in the second sum from the formula (12). Thus, we conclude that

$$
\frac{\partial f\left(x_{i}, u_{i}, t_{i}\right)}{\partial x_{i}} \cdot \Delta t+\lambda_{i}+\lambda_{i} \cdot \frac{\partial g\left(x_{i}, u_{i}, t_{i}\right)}{\partial x_{i}}-\lambda_{i-1}=0 .
$$

Dividing both sides by $\Delta t$ and taking into account the expression for $H(x, u, t)$ and the fact that $\frac{\lambda_{i}-\lambda_{i-1}}{\Delta t} \approx \dot{\lambda}\left(t_{i}\right)$, we conclude that

$$
\frac{\partial H}{\partial x}+\dot{\lambda}(t)=0
$$

This is equivalent to equation (7).

Thus, we get the desired derivation.

\section{Acknowledgments}

This work was supported in part by the National Science Foundation grants HRD-0734825 and HRD-1242122 (Cyber-ShARE Center of Excellence) and DUE-0926721, by Grants 1 T36 GM078000-01 and 1R43TR000173-01 from the National Institutes of Health, and by a grant N62909-12-1-7039 from the Office of Naval Research.

The authors are thankful to Anibal Sosa, Sungmi Lee, and to all the participants of the Conference on Applied Mathematics and Bioinformatics AMAB'2013 (Cali, Colombia, November 20-22, 2013) for valuable discussions. 


\section{References}

[1] R. Feynman, R. Leighton, and M. Sands, Feynman Lectures on Physics, Basic Books, New York, 2005.

[2] I. M. Gelfand and S. V. Fomin, Calculus of Variations, Dover, New York, 2000.

[3] I. M. Ross, A Primer on Pontryagin Principle in Optimal Control, Collegiate Publishers, San Francisco, California, 2009. 